Article

\title{
Energy Efficiency Perspectives of PMR Networks ${ }^{\dagger}$
}

\author{
Marco Dolfi, Simone Morosi *, Pierpaolo Piunti and Enrico Del Re \\ Department of Information Engineering, University of Florence, Via di Santa Marta 3, 50139 Firenze, Italy; \\ marco.dolfi@unifi.it (M.D.); pierpaolo.piunti@unifi.it (P.P.); enrico.delre@unifi.it (E.D.R.) \\ * Correspondence: simone.morosi@unifi.it; Tel.: +39-055-275-8547 \\ $+\quad$ This paper is an extended version of our paper published in Energy Efficient RRM Strategies for Current \\ and Upcoming TeTRA Cellular Systems. In Proceedings of the 2014 IEEE 10th International Conference \\ on Wireless and Mobile Computing, Networking and Communications (WiMob), Larnaca, Cyprus, \\ 8-10 October 2014; pp. 40-44.
}

Academic Editor: Willy Susilo

Received: 17 August 2016; Accepted: 16 December 2016; Published: 23 December 2016

\begin{abstract}
Recently, the concern about energy efficiency in wireless communications has been growing rapidly. Manufacturers and researchers have developed innovative solutions, highlighting the benefits in reducing operational expenditures (OPEX) and carbon footprint. Professional Mobile Radio (PMR) systems, like Terrestrial Trunked Radio (TETRA), have been designed to provide voice and data services to professional users. The energy consumption is one of the critical aspects of PMR broadband solutions and a major constraint for PMR services. The future convergence of PMR to the LTE system introduces a new topic in the research discussion about the energy efficiency of wireless systems. This paper focuses on the feasibility of energy efficient solutions for current and potentially future PMR networks, by providing a mathematical formulation of power consumption in TETRA base stations and assessing possible business models and energy saving solutions for enhanced mission-critical operations. The energy efficiency evaluation has been performed by taking into account the traffic load of a deployed TETRA regional network: in the considered network scenario with 150 base stations, significant OPEX savings up to 70 thousand Euros per year of operation are achieved. Moreover, the proposed solutions allow for saving more than 1 ton of $\mathrm{CO}_{2}$ per year.
\end{abstract}

Keywords: Terrestrial Trunked Radio (TETRA); Professional Mobile Radio (PMR); energy efficiency; cost reduction; economic considerations

\section{Introduction}

Professional Mobile Radio (PMR) systems represent a subset of mobile communications networks which are designed for mission critical communications. Since they provide a mobile wireless service to their users, as in the public radio network case, such systems are mainly addressed to public safety and security organisations to guarantee fail-safe and secure voice and data communications. On the other hand, the target requirements of the provided service are quite different than the public case, particularly in terms of system availability, security, resilience and reliability that are typical of mission critical communications [1].

Recently, PMR and public radio systems share common needs and interests. As a matter of fact, the possibility of providing broadband services is taken into account also by the PMR operators and manufacturers who are currently working on extending their systems capabilities. In order to increase the throughput and be able to support broadband services like video communications, Long Term Evolution (LTE) is now under investigation as the platform for future PMR systems [2].

Moreover, a greater sensibility toward environmental and energetic issues has been raised in research and the industrial world, and a lot of inherent international research activities have been funded [3-6]. The energetic costs are one of the major drivers of operational expenditures (OPEX) [7]. 
At the same time, controlling the cost of energetic waste allows for cutting greenhouse gas emissions, thus reducing air pollution with positive consequences on environment. An energy efficient cellular network can be achieved in multiple ways. First of all, an opportune deployment, taking into account the optimal site location, is needed to minimize the transmission power budget and the number of needed base stations. Moreover, considering that new technologies will be progressively introduced in an already deployed network, the hardware optimization is expected to significantly reduce the current energy waste, especially due to power amplifiers, the most power-consuming devices in a base station's equipment [8]. In this regard, replacing the old network devices with new and less power consuming ones will introduce a substantial energy efficiency gain. However, such energy gain should be further improved through radio resource management solutions aiming at optimizing the network usage with respect to the actual needs. As a matter of fact, the daily power consumption of a base station has a rather constant profile over time with respect to the transmitted traffic, as depicted in Figure 1. This behavior justifies the goal of using energy efficiency strategies to shape the power consumption to the actual energy demand. To this aim, many promising radio resource management strategies, mainly focused on power control and power amplifiers' sleep mode, have been proposed [9-12].

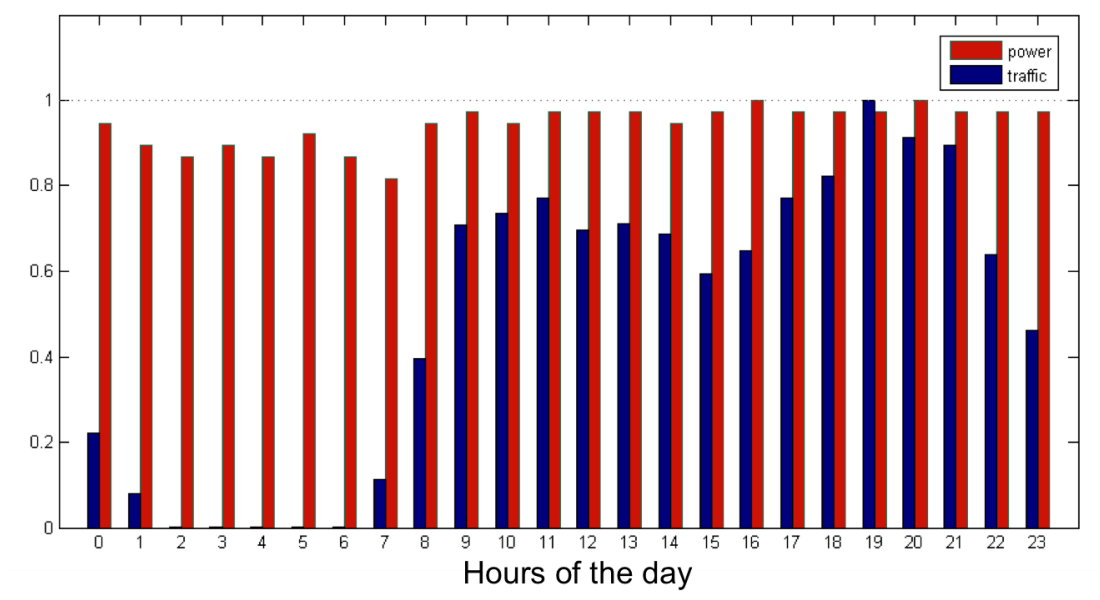

Figure 1. Power consumption and traffic of a generic public cellular base station during a day.

This paper discusses the feasibility of energy efficient solutions for current and future PMR networks. First of all, a review of the state-of-the-art of such systems is provided, mainly referring to the most popular system in Europe, which is Terrestrial Trunked Radio (TETRA). The main TETRA features are described highlighting the evolution to a broadband system through TETRA Enhanced Data Service (TEDS). Moreover, since the availability of IP broadband services will be a PMR target requirement, the feasibility of the transition to a TETRA over LTE system is discussed. In order to evaluate the energy impact of such a system, a TETRA/TEDS power consumption model is introduced, as well as some energy saving radio resource management techniques developed for commercial wireless systems. Then, an evaluation of the energy performance of an optimized TETRA/TEDS system has been done, also taking into account the potential energy efficiency gain, due to hardware improvements and radio resource management flexibility that will be allowed by the transition to an LTE infrastructure. Since the evaluation of power saving solutions should be based on actual network configuration and traffic load scenarios, this work extends the results presented in [13] by introducing a detailed energy consumption analysis focused on the traffic data of a deployed TETRA regional network. Finally, the performance of the considered systems is evaluated both from an economic and an environmental point of view, showing the positive impact of the energy management improvements in current TETRA/TEDS systems and the significant energy saving gain allowed by the LTE platform. The paper is organized as follows. Section 2 gives an overview of the state-of-the-art of TETRA system, 
describing its main features and discussing the feasibility of the potential future evolution over the LTE system. Section 3 introduces a power consumption model for today's TETRA systems, starting from the work carried out in literature about "green" cellular systems. Sections 4 and 5 describe some feasible strategies to increase the energy efficiency of current TETRA and TEDS systems and discuss the power saving gain, including environmental and economic effects, that can be obtained by actually implementing a TETRA over LTE infrastructure. Finally, Section 6 concludes the work.

\section{Present and Future PMR Systems}

PMR systems have been introduced to provide a speech and data two-way radio communication service in non-public networks tailored to the specific professional operational needs. Typically, the main users of such kind of services are military, police and public safety forces, but also private institutions like transportation and logistic companies. The target PMR scenario is represented by the mission critical communications. It is characterized by four key requirements: resilience and highly availability of the infrastructure, reliability of the communications, security and possibility of point-to-multipoint communications to support group calls and messages. A resilient and highly available infrastructure can be obtained by redundant network architecture and fail-safe network elements, allowing a minimum service even if the connection to the infrastructure is not possible or limited. Moreover, the communication is considered reliable if the provided service is accessible and stable. Therefore, the target quality of service must be reached within the entire coverage area and the communication setup must be extremely fast. Security is needed to protect users from malevolent actions like jamming, interception and spoofing, providing features like mutual authentication of terminals, jamming compensations, end-to-end encryption and temporarily terminal disabling. Low power consumption is also needed to reduce the operational costs. Such requirements have been largely implemented in the mobile radio terminals by introducing, for instance, transmission power control in order to increase the battery lifetime [14]. Currently, TETRA $[15,16]$ is the most widely used PMR standard, providing a reliable infrastructure to support mission critical communications. As depicted in Figure 2, the PMR evolution, particularly referring to TETRA, is linked to the commercial radio system evolution since PMR manufacturers cannot afford in-depth research and development budgets to develop next generation PMR mobile radio technologies in parallel to or even ahead of commercial mobile radio manufacturers. Therefore, since its beginning, PMR systems have been exploiting commercial radio systems technologies and standards. The remainder of this section describes the TETRA evolution, starting by the original TETRA system and TETRA Enhanced Data Service (TEDS), representing the state-of-the-art, and a possible evolution related to the broadband services provided by the $4 \mathrm{G}$ commercial standard, identified as TETRA over LTE.

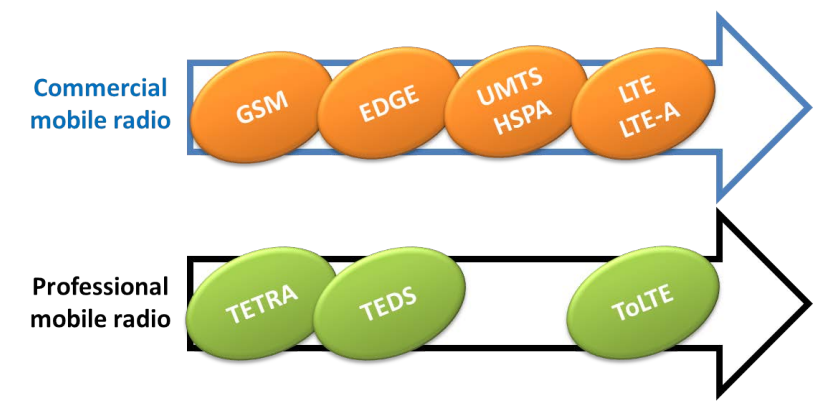

Figure 2. Terrestrial Trunked Radio (TETRA) evolution.

\subsection{TETRA and TEDS for Speech and Data Communications}

TETRA is a multiple access digital system for secure private radio communications. It allows the transmission of high quality voice and low-speed data, and it has been proposed mainly for 
emergency services, public safety, and, in general, for all the scenarios where a bounded secure area for communications is needed.

The TETRA system has some unique functionalities that cannot be obtained by commercial cellular systems: these features are motivated by the specific purposes of the secure private radio systems with respect to those pursued by widespread cellular technologies. In particular, TETRA offers:

- group calls;

- $\quad$ reduced call set-up time (below $300 \mathrm{~ms}$ ) with respect to 2G technologies;

- $\quad$ direct mode of operation using other mobile devices as repeaters;

- $\quad$ secure data transmission by end-to-end encryption;

- push-to-talk mode.

As depicted in Figure 3, the TETRA architecture is similar to a generic cellular network and includes the following standard interfaces:

- Air Interface (AIR I/F), which ensures the interoperability of terminal equipment of different manufacturers;

- Terminal Equipment Interface (TEI) facilitating the independent development of mobile data applications;

- Inter Systems Interface (ISI), which allows the interconnection between TETRA networks of different manufacturers;

- Direct Mode Operation (DMO) guaranteeing the communication between terminals also beyond network coverage.

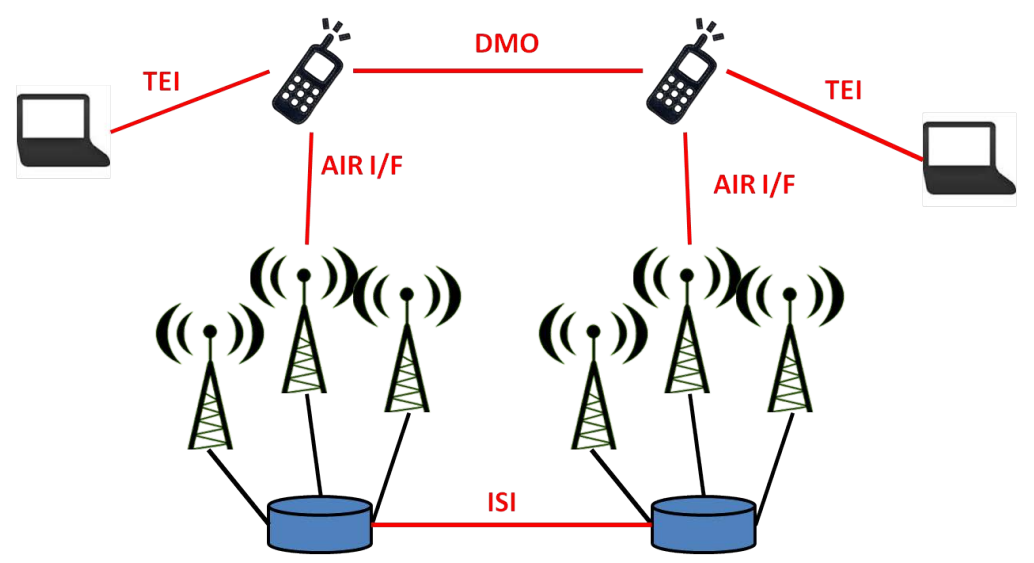

Figure 3. TETRA architecture.

A Time Division Multiple Access (TDMA) is used to allocate four time slots, each of which has a duration of $14.167 \mathrm{~ms}$, into a $25 \mathrm{kHz}$ bandwidth carrier. Each time slot represents a full rate channel or, optionally, two half rate channels in order to increase the random access. Frequency division duplexing is used to associate to a time slot the uplink and downlink channels. Data and control information is mapped within each channel and the transmission is done through $\pi / 4$-PSK modulation. The physical content of a time slot is organized as a burst, as described in Figure 4. At each base station, the first slot of each TDMA frame of one carrier is occupied by the Broadcast Common Channel (BCCH). The remaining time slots of such carriers and, eventually, all the time slots of other available carriers can be assigned to a Traffic Channel (TCH). When a channel is idle because it is not assigned to any $\mathrm{TCH}$, a dummy burst is transmitted in order to maintain a continuous bit flow. 


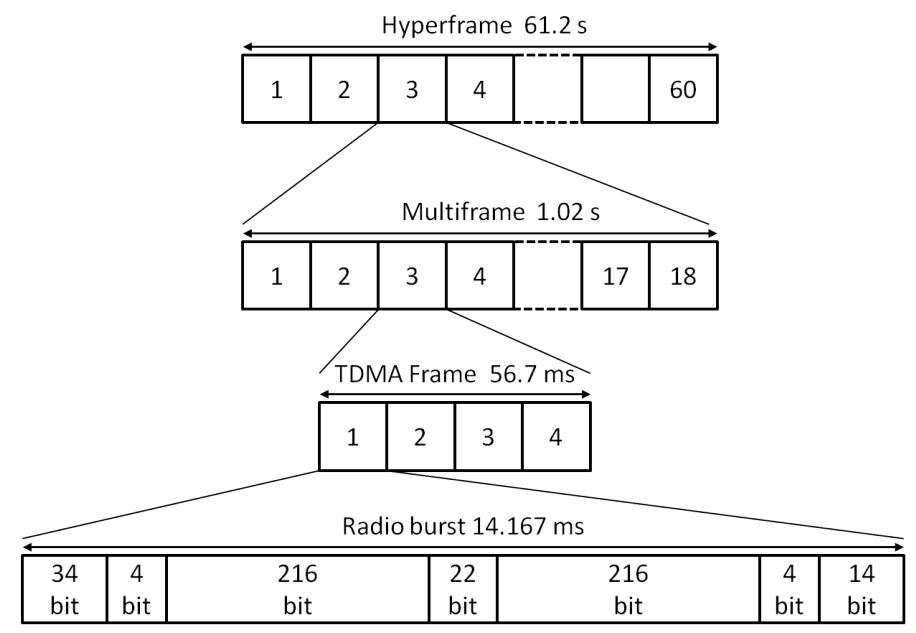

Figure 4. TETRA radio frame.

The first release of TETRA allows a maximum uncoded data rate of $28.8 \mathrm{kbps}$. In order to improve this standard to support IP-based multimedia services, TEDS has been introduced taking care of the maximum backward compatibility [17]. TEDS can be considered as an evolution of TETRA system, with improvements focusing on transmission and bandwidth extension, in order to meet the need for higher speed data services. In particular, as reported in Figure 5, channels with different bandwidths are available. Allowed bandwidths are $25 \mathrm{kHz}$, as in the previous release, $50 \mathrm{kHz}, 100 \mathrm{kHz}$ and $150 \mathrm{kHz}$. Moreover the adoption of spectral-efficient multilevel modulation schemes enables the possibility of link adaptation: 4-QAM, 16-QAM and 64-QAM are added to $\pi$ /4-PSK scheme, used in the first release of TETRA. In order to detect errors and protect the information, different channel coding schemes have been introduced. By adaptively selecting the opportune modulation, taking into account the needs and the link quality, a throughput beyond $500 \mathrm{kbps}$ can be obtained, as shown in Table 1 . The TEDS improvements are not only related to physical layer. Classifying data flows into classes allows for negotiation of the opportune quality of service for each flow in terms of throughput, delay, precedence and reliability. Allowed classes are real-time class for live audio and video transmission, and telemetry class for bursted low capacity transmissions, and background class for file transfer and web applications.

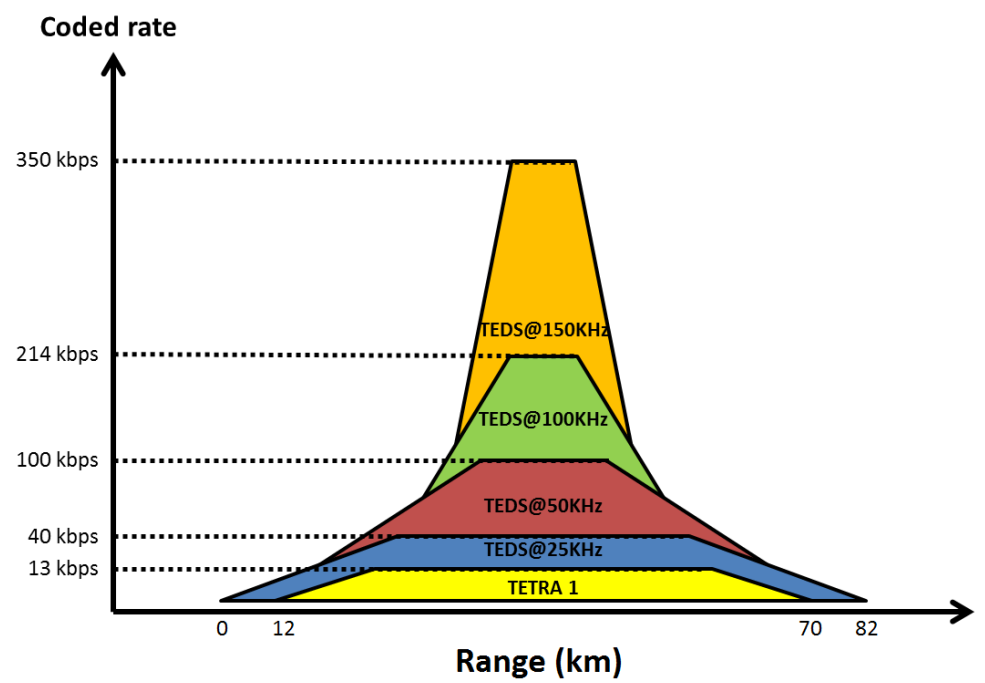

Figure 5. TETRA Enhanced Data Service (TEDS) bandwidth and performance. 
Table 1. Channel coding and TETRA Enhanced Data Service (TEDS) throughput.

\begin{tabular}{|c|c|c|c|c|}
\hline & $25 \mathrm{kHz}$ & $50 \mathrm{kHz}$ & $100 \mathrm{kHz}$ & $150 \mathrm{kHz}$ \\
\hline B $/ 4$-DQPSK, $r=2 / 3$ & $15.6 \mathrm{kbps}$ & & & \\
\hline B $/ 8$-D8PSK, $r=2 / 3$ & $24.3 \mathrm{kbps}$ & & & \\
\hline 4-QAM, r = 1/2 & 11 kbps & $27 \mathrm{kbps}$ & $58 \mathrm{kbps}$ & $90 \mathrm{kbps}$ \\
\hline 16-QAM, r = 1/2 & $22 \mathrm{kbps}$ & $54 \mathrm{kbps}$ & $116 \mathrm{kbps}$ & 179 kbps \\
\hline 64-QAM, r = 1/2 & $33 \mathrm{kbps}$ & 80 kbps & $175 \mathrm{kbps}$ & $269 \mathrm{kbps}$ \\
\hline $64-Q A M, r=2 / 3$ & $44 \mathrm{kbps}$ & $107 \mathrm{kbps}$ & $233 \mathrm{kbps}$ & $359 \mathrm{kbps}$ \\
\hline 64-QAM, r = 1 & $66 \mathrm{kbps}$ & $160 \mathrm{kbps}$ & 349 kbps & $538 \mathrm{kbps}$ \\
\hline
\end{tabular}

\subsection{The PMR Evolution through the LTE System}

Currently, most TETRA manufacturers are looking to the future by implementing a TETRA over LTE system $[18,19]$ in order to provide higher data rate and lower latency services.

LTE is the state-of-the-art standard for commercial mobile communications, providing an all-IP connectivity through an infrastructure designed for very high speed services.

Even if LTE has been developed for commercial use, some work groups in 3GPP have been working on the adaptation of the standard to mission critical communications [20]. In particular, the following items are currently under investigation:

- Group communication and push-to-talk (PTT),

- Proximity based services,

- Network resilience,

- High power user equipments,

- Enhanced Radio Access Network (RAN) sharing,

- Priority and Quality of Service (QoS) control.

More specifically, group communications, PTT and proximity based services, that enable device-to-device (D2D) communications, are the key requirements for public safety mission critical voice services. The work on such subjects has been carrying on within the LTE Release 12 and LTE Release 13 groups [21-23]. In the framework of these releases, some issues about resilience and RAN sharing are also being discussed. For example, in order to face a disaster causing the failure of some devices, any base station should be able to act alone in connecting the served users with the rest of the network [24]. Moreover, an enhanced flexibility in sharing network resources could allow the adoption of smart radio resource management strategies between critical and non-critical users [25]. As for high power user equipment and priority and QoS control, such features are already provided by the LTE technology and the work that is being carried on is related to future enhancements [26,27].

Whilst these outcomes will clearly directly affect infrastructure vendors, the market for user equipment, applications and other end-user equipment, and services can be expected to be less impacted. In other words, whether or not the bearer network is owned and operated by the user organization, or services are provided by mobile network operators, users will still require terminal devices and applications. In a standardized market, competition and innovation, as well as economies of scale resulting from the wider global LTE user equipment ecosystem, can be expected to influence prices. A strong standards-based approach will ensure interoperability between different vendors leading to a competitive equipment market.

Besides the opportunities offered by exploiting the LTE technology for PMR services, the main issue resides in the way such services should be provided. Since there is no commercial interest to develop and integrate all the PMR functionalities over the LTE infrastructure, especially the ones related to security end encryption operations, only private networks should be preferred. Private networks could be self-deployed by the interested organizations for its own activities or 
provided by a third-party. In both cases, once the LTE system will be compliant with mission critical communications requirements, the main open issue will be the actual channelization, since LTE is designed to operate with bandwidths starting from 1.4 MHz. However, the PMR evolution over the LTE system, i.e., TETRA over LTE, represents a big opportunity for current PMR users to increase the efficiency of their own spectrum usage.

\section{Modelling Power Consumption in PMR Systems}

A TETRA base station is composed of several functional blocks that are depicted in the diagram of Figure 6. In particular, the main blocks are baseband unit (BB), radio frequency unit (RF) and power amplifier (PA), which together form the transmission chain. Moreover, the site control unit (SCU) is responsible for the management operations of the considered site. In order to maintain the opportune temperature for the whole site, a cooling system is considered. This system is responsible for cooling down the site environment coping with the temperature increase due to electronic operations, or, conversely, provides a heating function when the devices temperature gets below the operational target. Transformers and rectifiers are needed to adapt the network power in order to feed the base station devices, and their operation is considered in the main supply system.

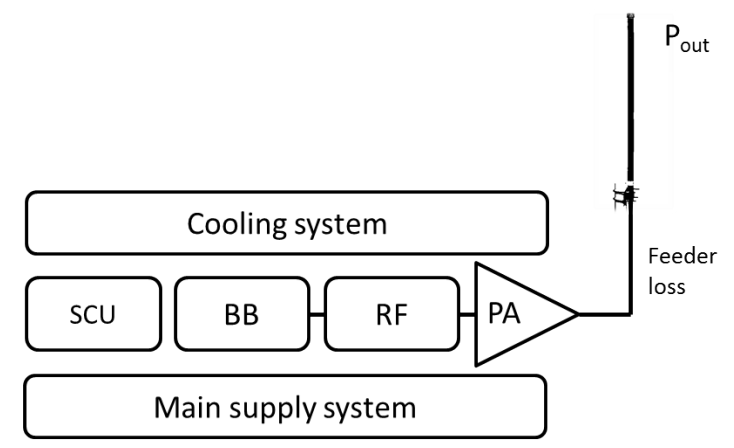

Figure 6. TETRA base station model.

A TETRA base station power consumption model can be obtained by adapting the one proposed in [28]. The following equation is considered:

$$
P_{\text {in }}=N_{T R X} \frac{\frac{P_{\text {out }}}{\mu_{P A}\left(1-\sigma_{\text {feed }}\right)}+P_{R F}+P_{B B}+P_{S C U}}{\left(1-\sigma_{D C}\right)\left(1-\sigma_{M S}\right)\left(1-\sigma_{\text {cool }}\right)},
$$

where $N_{T R X}$ is the number of transceivers at the base station; $P_{\text {out }}$ is the transmission power; $P_{B B}, P_{R F}$ and $P_{S C U}$ are, respectively, the power spent for the base band operation, the RF stage and the site control unit. The feeder loss is modeled by the $\sigma_{\text {feed }}$ parameter and $\mu_{P A}$ is the power amplifier efficiency. Finally, $\sigma_{D C}, \sigma_{M S}$ and $\sigma_{c o o l}$ model the DC loss, i.e., the loss in the transmission chain, the loss in the main supply system and the loss for cooling. The power consumption parameters are summarized in Table 2. Equation (1) shows that the power consumption in base stations can be divided into a static component and a dynamic component. In particular, the dynamic component is related to the output power: by varying $P_{\text {out }}$, for instance when managing different amounts of traffic, and then the variable part of the total power consumption changes. On the other hand, the static component, represented by $P_{B B}, P_{R F}$ and $P_{S C U}$, does not significantly change for different $P_{\text {out }}$ values, or the variations are negligible. Therefore, the model can be simplified as follows:

$$
P_{\text {in }}=N_{T R X}\left(\Delta_{p} P_{\text {out }}+P_{0}\right),
$$

where the PA efficiency and losses effects are included in $\Delta_{p}$ and $P_{0}$ takes into account the static BB, 
RF and SCU power consumption. By considering the parameters in Table 2, in this paper, $\Delta_{p}=7.5$ and $P_{0}=280 \mathrm{~W}$ have been assumed.

Table 2. TETRA base station power consumption parameters.

\begin{tabular}{ccc}
\hline Transmission power & $P_{\text {out }}$ & {$[0,20] \mathrm{W}$} \\
Power Amplifier (PA) efficiency & $\mu_{P A}$ & 0.25 \\
Radio Frequency (RF) power consumption & $P_{R F}$ & $114 \mathrm{~W}$ \\
Baseband (BB) power consumption & $P_{B B}$ & $75 \mathrm{~W}$ \\
Site Control Unit (SCU) power consumption & $P_{S C U}$ & $23 \mathrm{~W}$ \\
DC loss & $\sigma_{D C}$ & $7.5 \%$ \\
Feeder loss & $\sigma_{f e e d}$ & $50 \%$ \\
Main supply loss & $\sigma_{M S}$ & $9 \%$ \\
Cooling loss & $\sigma_{\text {cool }}$ & $10 \%$ \\
\hline
\end{tabular}

\section{Energy Efficiency Strategies for PMR Systems}

\subsection{Carrier Sleep Mode}

Because of the impact of fixed power consumption, which is the power spent by the base station even for zero transmission power, the most common energy saving strategy proposed for commercial cellular system is the carrier sleep mode. As a matter of fact, in order to improve the capacity, operators could deploy more than one carrier per cell. As explained in Section 2, the cell signaling is transmitted just over one carrier, while the other ones only manage the traffic and the dedicated signaling. Therefore, carrier sleep mode works automatically by deactivating the unused carriers; the only carrier that cannot be ever deactivated is the one carrying the cell signaling in order to maintain the coverage of the area. Deactivating a carrier, or putting it on sleep mode, means putting the carrier in a low power consumption state, such that the base station controller can make it operating when needed in a very small time (a few seconds).

By adopting the carrier sleep mode, the power consumption model presented in Equation (2) can be modified as follows:

$$
P_{i}= \begin{cases}\Delta_{p} P_{\text {out }}+P_{0}, & \text { if the } i \text {-th carrier is ON, } \\ P_{\text {sleep }}, & \text { if the } i \text {-th carrier is sleeping, }\end{cases}
$$

where $P_{\text {sleep }}$ is the power consumption of the sleep mode state. In this study, $P_{\text {sleep }}=140 \mathrm{~W}$ has been assumed.

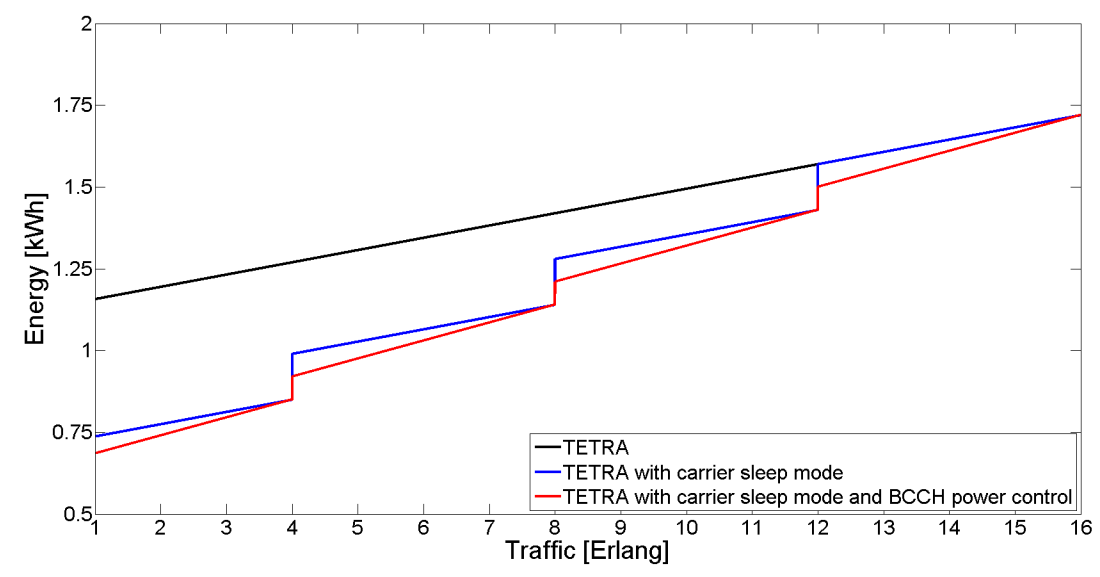

Figure 7. Energy consumption versus traffic for TETRA. 
The performance of TETRA carrier sleep mode is depicted in Figure 7, which shows the energy consumption of a TETRA base station that is equipped with four carriers as a function of traffic load: the carrier sleep mode, as expected, provides significant energy savings, especially in the case of low traffic load, converging instead to the baseline consumption with peak traffic load.

\subsection{BCCH Carrier Power Control}

Power control is a software based solution that introduces an energy saving mode on the BCCH transceiver. Such a solution reduces the overall power consumption by transmitting dummy bursts on the idle channels, i.e., on the time slots that are not allocated to a $\mathrm{TCH}$, with a power level lower than the maximum transmission power of the $\mathrm{BCCH}$ channel. The behaviour of power control is shown in Figure 8: note that, in our analysis, a $2 \mathrm{~dB}$ power reduction has been considered. In order to keep the cell range unaltered, the $\mathrm{BCCH}$ channel is always transmitted at full power. The introduction of power control allows the power consumption to vary according to the served traffic. Figure 7 shows the behavior of a TETRA base station power consumption when power control is applied. The largest energy saving is achievable in the case of low traffic load, while for higher traffic load values, the performance of power control converges to the TETRA baseline case. We observe that the gain due to power control on the $\mathrm{BCCH}$ carrier is smaller with respect to the impact of carrier sleep mode. Therefore the combined adoption of the two solutions should be supported. In this regard, the proposed power consumption model has been evaluated by statistical analysis, considering a weekly traffic data set of a deployed TETRA regional network and a 95\% confidence interval.

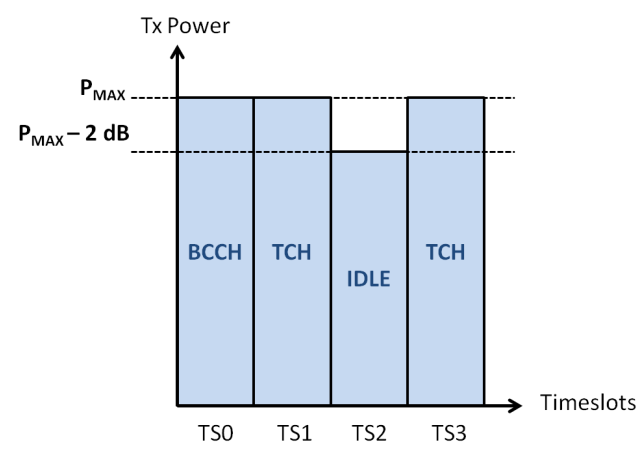

(a) Power control

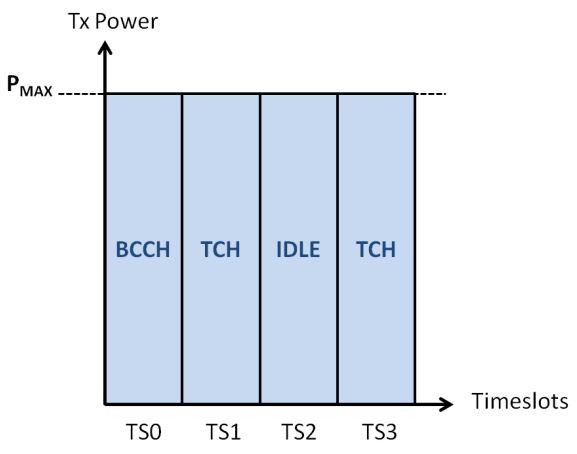

(b) No power control

Figure 8. Transmitted signals for power control (a) and no power control (b) cases.

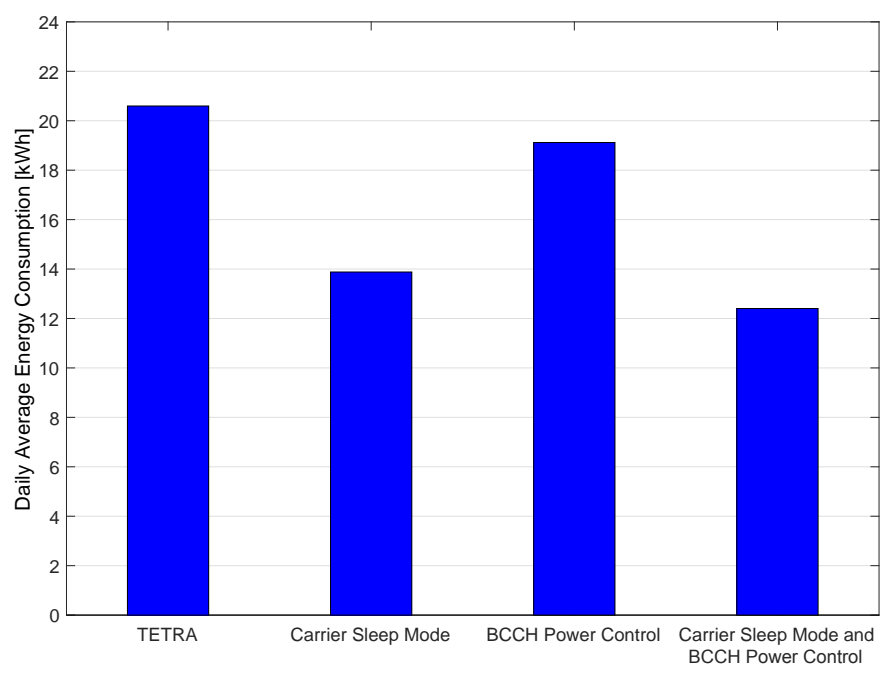

Figure 9. Daily average energy consumption of a single TETRA base station. 
Figure 9 shows how the considered strategies perform in terms of daily average power consumption. As expected, the application of the carrier sleep mode brings significant energy savings, resulting in a 30\% lower power consumption, considering the daily average traffic load of a single TETRA base station. Even if the energy gains achieved by power control on the BCCH carrier are smaller, employing this strategy in combination with the carrier sleep mode results in an additional $10 \%$ saving, showing that the common techniques proposed for commercial cellular standards could actually improve the energy efficiency of PMR systems.

\section{Technical and Economic Considerations on Energy Efficiency of Next Generation PMR Systems}

\subsection{Business Models for Mobile Broadband PMR}

When evolving to mobile broadband communications, public safety agencies may choose from numerous business models that will support its specific needs, taking into account existing PMR network operations, available spectrum, regulatory environment and financial resources. It may contract services provided by a mobile network operator (MNO), operate or use a service from a dedicated virtual network over a mobile operator's infrastructure (G-MVNO), build a wholly owned and operated dedicated network, or use a mix of different approaches [29]. Adding LTE mobile broadband capabilities to existing PMR networks in a nondisruptive, cost-effective and energy-efficient way can be complex, with many factors to consider. These include startup costs, operating and capital expenditures (OPEX and CAPEX), expected revenues, available spectrum, existing network equipment, commercial wireless services and the political environment. The following are five possible business models that are proposed to match the trade-off between objectives and constraints.

\subsubsection{Contract Services through an Existing Mobile Network Operator}

In this model, the public safety agency simply contracts data subscriptions with an MNO to provide mobile broadband services. Public safety users and consumers share the same spectrum and network. The public safety entity pays a consistent, predictable periodic fee for network access, usually a function of some known factor, such as the number of end users, devices or usage. This arrangement is relatively inexpensive if traffic, the number of users and subscription fees can be low, and fast if MNO LTE service already is available. CAPEX concerns only applications and terminals, which could remain significant if a large number is required. OPEX consists mainly of monthly fees for using the MNO service, and is proportional to the number of users and usage volume. Challenges for the MNO model include no control over four critical requirements: coverage (usually very poor in sparsely populated areas), availability, prioritization and resilience. Typically, little or no support exists for mission-critical features, and gaps in coverage can occur where the population density is low, such as in rural and isolated areas. For mission-critical needs, these issues might be addressed through stringent service level agreements (SLAs) to assure such features as priority access or network redundancy in case of an outage, which may significantly increase the subscription fee. In addition, most MNOs have a monthly data cap and additional fees for excess usage, which can significantly impact OPEX.

\subsubsection{Obtain Service from a Government Mobile Virtual Network Operator}

The MVNO approach has become prevalent in the commercial sector, where branded operators resell bulk-purchased wireless services to consumers while providing their own usage plans, billing and customer support. The MVNO approach can be extended to the support of public safety users. In that case, the MVNO, called a G-MVNO (Government MVNO) provides added-value services (such as user and device management, customer care, end-to-end security, billing and so on) to the public safety users that, in turn, get access to secure broadband data services when the G-MVNO leverages the $4 \mathrm{G}$ access network from the MNO. The G-MVNO model offers more control over services and security than the MNO approach, providing a ready-made network for basic public safety needs. It keeps CAPEX moderate (mainly terminals and a few LTE core network nodes). A G-MVNO can 
manage services and management over a mix of 4G, 3G and PMR platforms for the best possible availability in routine situations and major crisis. It can be configured to combine security, availability, ease of use and economics tailored for public safety, while keeping the effective management of end-to-end data service a first priority. About the cons of this approach, as with the MNO model, a G-MVNO provides no absolute control over coverage (especially in rural areas), availability and assured resilience. In addition, depending on the MNO, it may offer limited support of some critical public safety features, such as direct mode or group calling.

\subsubsection{Deploy Dedicated Network Services through a Public-Private Partnership Project}

The Public-Private Partnership Project (PPP) business model features a dedicated and standalone LTE network, which is deployed, operated and maintained by an MNO and/or another independent operator. This type of network is typically owned by a telecom operator, which provides the service to the public safety agencies while usually assuming the financial, technical and operational risk of the service offer. One of the key benefits of the PPP model is that the public safety agency is the only entity using the network. CAPEX and OPEX can be reduced through synergies in the reuse of antenna sites, backhaul and technical skills contributed by the private partner. Public safety communications requirements are assured and customized, with full control over such critical specifications as latency, coverage and resilience. The main challenge is that this model requires having access to a dedicated broadband public safety spectrum and negotiating with a partner to invest the upfront CAPEX to build the network. However, many synergies can exist to minimize this upfront investment.

\subsubsection{Build, Own and Operate a Dedicated Private Network}

In this model, the public safety agency finances, procures, builds and manages its own network, setting technical requirements for capacity, security, reliability, redundancy and robustness. It takes full responsibility for all network elements and software, and employs in-house personnel to build, manage, operate and maintain the network. The extent of upfront costs depends on the scale of deployment (local, regional or national), whether the network is shared among several entities and/or whether the deployment is scheduled gradually over years or within a shorter time period. The clear advantage with a dedicated LTE network is that it can be designed to match all mission-critical requirements, with the agency having full control over the procurement process. Specifications (such as site hardening, extended coverage and resilience to multiple faults, extreme events, and energy saving solutions) can be tailored to missions, as well as to the agency's future evolution strategy. As with the PPP model, a dedicated network can offset CAPEX and OPEX by operating as a wholesaler where regulations permit, or sharing with other critical users of the public sector such as defense, utilities and transportation agencies. On the other hand, specifying, building and maintaining a dedicated network requires significant upfront investments and technically skilled professionals for network operations. Acquiring dedicated LTE spectrum may require an initial investment or an annual fee. A dedicated spectrum must be cleared of any previous service, usually a slow process in countries where no dedicated spectrum currently exists. Both CAPEX and OPEX typically could be higher in this model. However, CAPEX depends significantly on the spectrum of operations (the lower the spectrum, the lower the number of sites to deploy) and can be planned over multiple years to deploy in critical areas first.

\subsubsection{Combine a G-MVNO with a Private Network (Hybrid Model)}

Given that the spectrum is a scarce resource in many regions, some agencies may elect to build a custom communications network dedicated exclusively to mission-critical services, while conducting less critical back-office operations through commercial operators using the G-MVNO model. This approach can be implemented relatively easily, since LTE is both a technology for commercial carriers as well as the new-generation platform for PMR. This model has the advantage of rapid deployment without having to wait for dedicated broadband spectrum to become available. 
It enables an agency to handle very high volumes of everyday data traffic while preserving a fully controlled, mission-critical core for emergency situations. It is flexible and positions the agency for future evolution to a fully dedicated network. It allows agencies to make investment efficient by combining a mix of commercial and dedicated spectrum terminals. In addition, when both options are available, the MNO network can be used to offload traffic from the private network that is not mission-critical, preserving key resources for mission-critical traffic and applications. Following this strategy means less than full control over the entire network and its coverage area, and may require mobile transmitters or antennas on some occasions. Developing a hybrid approach also introduces slightly greater complexity to design, operations and financial models, requiring critical consideration and coordination of these elements.

Public safety agencies embarking on an LTE deployment project must consider factors such as budget, regulatory issues, internal resource constraints, coverage and reliability targets, available spectrum (frequency band and bandwidth) and number of end users when choosing the best overall design and business model. Agencies should also have a plan for energy management because the wealth of robust data and growth of mobile traffic will offer new ways of conducting operations. Regardless of the model chosen, the network must be defined through an end-to-end service-centric approach.

\subsection{LTE Energy Efficiency Gain}

The actual transition from the traditional TETRA infrastructure to the LTE platform could introduce a significant energy saving gain without considering the adoption of any particular strategy. Referring to the most power consuming stage, that is the RF stage, the main difference between TETRA and LTE is related to modulation types and techniques. In particular, TETRA, as all the systems coming out from 2G cellular technologies, employs constant envelope modulations, which improve the efficiency of power amplifiers thanks to the low peak to average power ratio (PAPR). Recently, several techniques have been proposed in order to increase the PA efficiency. In particular, Envelope Tracking has been found to be the most effective one and has been included in the LTE standard. Envelope Tracking dynamically adjusts the supply voltage to the envelope of the RF input, allowing a better efficiency also for high PAPR modulations, like Orthogonal Frequency-Division Multiplexing (OFDM) used in the LTE system. Looking at the power consumption model, a comparison could be done considering the LTE system reference case provided by the European Seventh Framework Programme (FP7) EARTH project [30]. The reference case has been obtained from the power measurements of a $10 \mathrm{MHz} 2 \times 2$ LTE base station, and, referring to the linear power model in Equation (2), $\Delta_{p}=4.7$ and $P_{0}=130 \mathrm{~W}$ have been set.

\subsection{Radio Resource Management Strategies for Energy Efficiency}

Looking at commercial cellular networks, the adoption of efficient radio resource management solutions is one of the most effective ways to reduce the overall energy consumption. As a matter of fact, network dimensioning is peak-load oriented. Therefore, most of the day the traffic is much lower than in peak hours, and a lot of energy gets wasted. The main goal of an energy efficient radio resource management scheme is to adapt the network energy consumption to the actual daily traffic load. The main way to adapt the radio resources to the users' requests is the introduction of base station sleep mode, which gives the system the possibility to put some devices of a base station in a low power state. Referring to the LTE system, several sleep mode techniques have been proposed [10]. In particular, frequency domain, system domain and time domain approaches are under investigation in current research about green wireless access networks. All the solutions focus on putting the RF power amplifiers on low consumption state. Frequency domain solutions are able to manage the available bandwidth at the base station by putting on sleep mode the relative carrier blocks. In order to maintain the same power spectral density, the reduced bandwidth requires less radiated power. Spatial domain solutions derive from the coexistence of multiple radio access technologies, which are allowed by the LTE standard: the global network energy efficiency can be improved by 
introducing cooperation schemes between the available access technologies. Other spatial domain solutions are reducing antenna number at the base station and dynamically configuring cells in a multicell scenario. Even if such approaches are an attractive solution for commercial LTE cellular networks, characterized by the densification of base stations and cell layers, they are not suitable for PMR networks like TETRA. Therefore, in order to design an energy efficient TETRA over LTE network, just the time domain approaches should be investigated. The most promising time domain solution is identified as cell discontinuous transmission (cell DTX), a hardware feature based on the deactivation of some components of a base station when there is no traffic, i.e., during the zero transmission time intervals [31]. As we observed before, if a base station with no traffic can be put into sleep mode, then the idle power consumption will be significantly reduced; in addition, cell DTX acts only when no data or signaling is transmitted, thereby the cell coverage is not affected. As shown in Figure 10, only a small fraction of each subframe must be transmitted even if the base station is not managing any traffic flow. In particular, a high cell DTX gain can be obtained using Multicast-broadcast single-frequency network (MBSFN) or extended cell DTX subframes instead of the normal unicast ones [10].

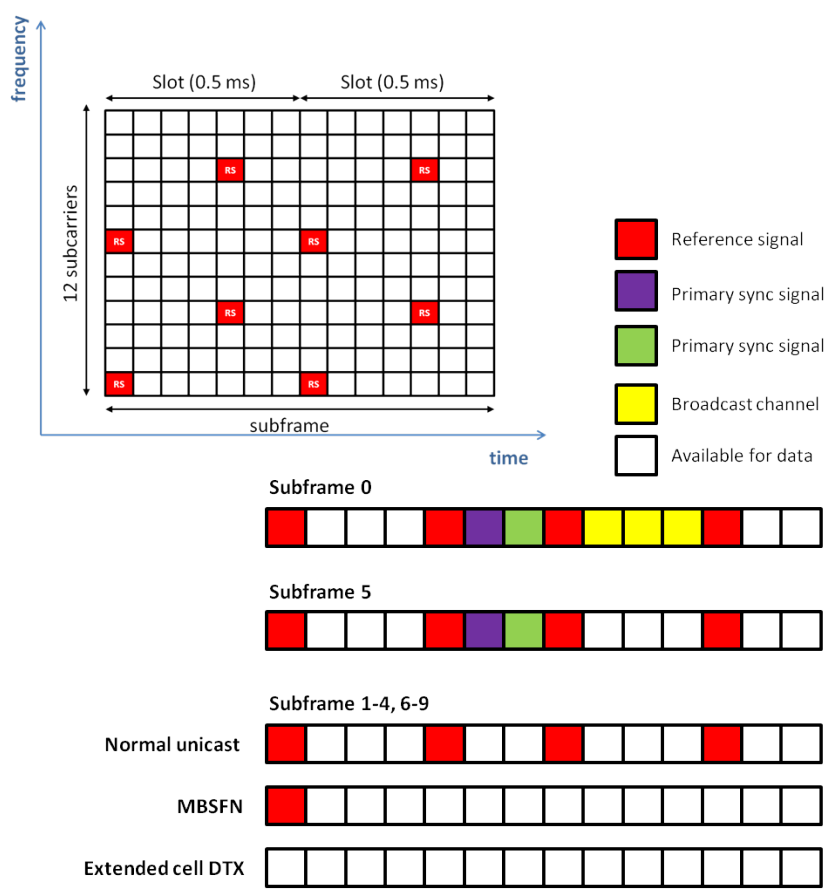

Figure 10. Cell DTX: power amplifiers can be put in low power state if there is no downlink traffic.

Unlike long term sleep solutions, cell DTX deactivates only some parts of the base station equipment, in order to ensure the immediate activation of the base station upon request. This approach hence allows the energy consumption to adapt to the variation of traffic in a very short time scale. Thanks to these characteristics, cell DTX is able to decrease the baseline power consumption of the base station to $P_{s}=\delta P_{0}$, where $0 \leq \delta \leq 1$. The power consumption model presented in (2) then can be written as follows:

$$
P_{\text {in }}=N_{T R X}\left(\Delta_{p} P_{\text {out }}+(1-\delta) \eta P_{0}+\delta P_{0}\right) \text {. }
$$

Here, $\eta$ denotes the load of the base station, while $(1-\delta) \eta P_{0}$ represents the load dependent baseline power consumption, bound to the fast traffic adaptation property of cell DTX. Note that, in the case of $\delta=1$, the base station does not have the DTX capability, and the power consumption model is the same as the one observed in Equation (2). To compare the energy efficiency performance of the advanced TETRA systems and the $4 \mathrm{G}$ commercial standard LTE, we consider a volume of traffic 100 times greater than the daily average traffic load of a current TETRA base station. Figure 11 
displays the performance of the described energy efficiency solutions for TEDS and LTE systems, in terms of daily energy consumption of a single base station. We consider TEDS systems with $50 \mathrm{kHz}$ and $150 \mathrm{kHz}$ bandwidths and assume $\delta=0.1$ for the case of LTE with cell DTX. We observe that the combination of carrier sleep mode and power control on the BCCH carrier can bring striking energy savings for TETRA network, although the energy efficiency performance of such systems is very far from the performance of the current LTE commercial standard. Despite the high hardware efficiency of LTE system, the introduction of cell DTX helps to further break down the energy consumption of the network by significantly reducing the baseline power consumption of the base stations. Looking at the PMR broadband evolution over the LTE system, such a feature represents a very promising energy saving approach, considering the typical low traffic density of PMR systems, compared to cellular public radiotelephone standards.

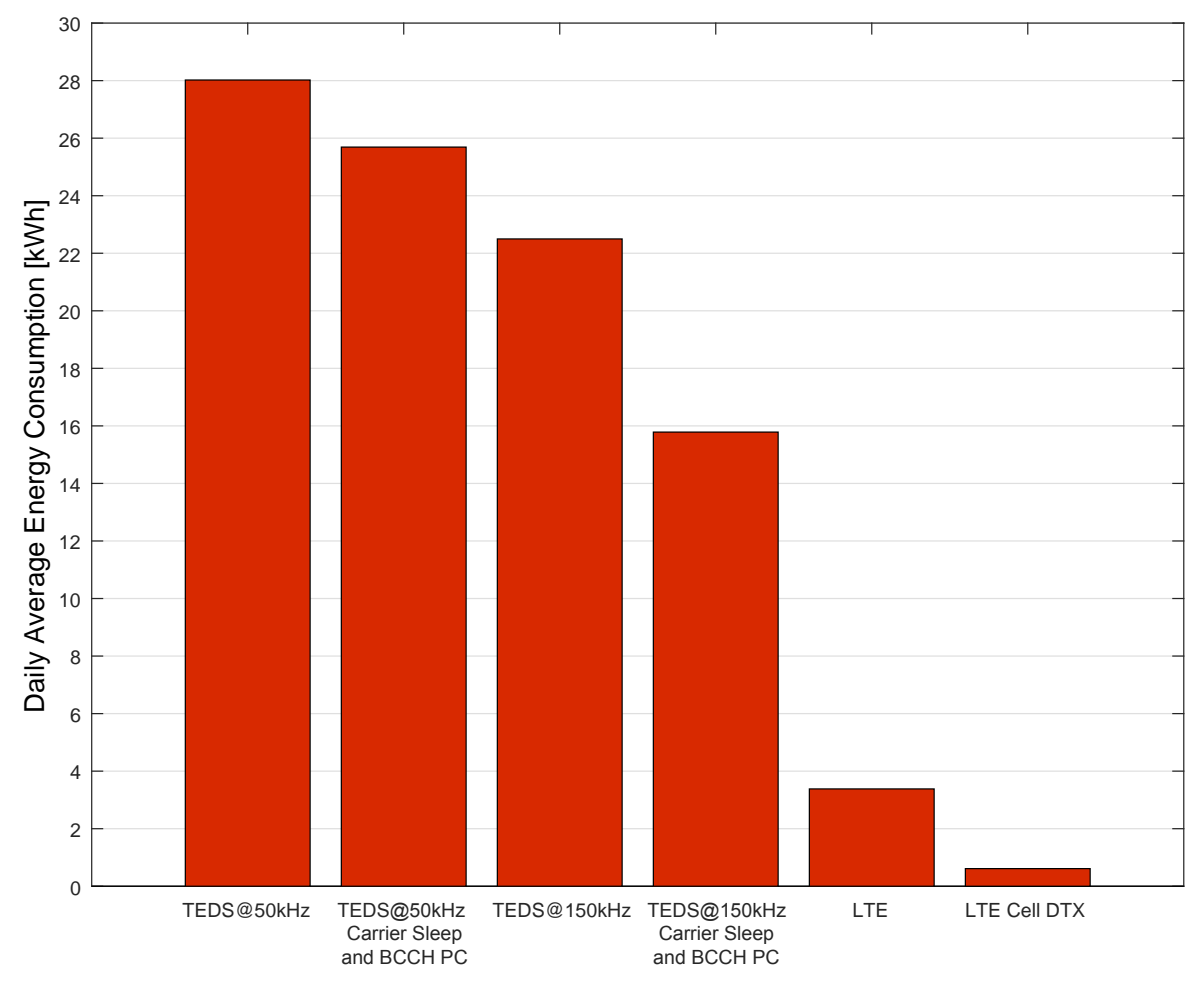

Figure 11. Comparison of daily average energy consumption of TETRA and LTE radio base stations.

From an economic perspective, the results presented in Figure 12 show the impact of the power saving features in terms of annual OPEX, considering the scenario of a regional network with 150 base stations and an energy cost of 0.20 Euros per $\mathrm{kWh}$. Adopting advanced energy efficiency strategies in the TETRA network results in significant OPEX savings of up to 70 thousand Euros per year of operation, compared to the current standard technology; this positive trend is also considerable with the introduction of highly efficient cell DTX solutions in LTE networks. Regarding the sustainable development of modern communications systems, the overall network energy saving also guarantees a remarkable reduction in terms of carbon emission of $\mathrm{CO}_{2}$ : considering an average carbon emission of $525 \mathrm{~kg}$ of $\mathrm{CO}_{2}$ per $1000 \mathrm{kWh}$, the proposed energy efficiency solutions can save more than 1 ton of $\mathrm{CO}_{2}$ per year in advanced TETRA networks. In the case of LTE systems, the adoption of cell DTX has an equally great impact, by granting more than $80 \%$ reduction of annual carbon emission of $\mathrm{CO}_{2}$, with respect to the standard LTE network configuration. 


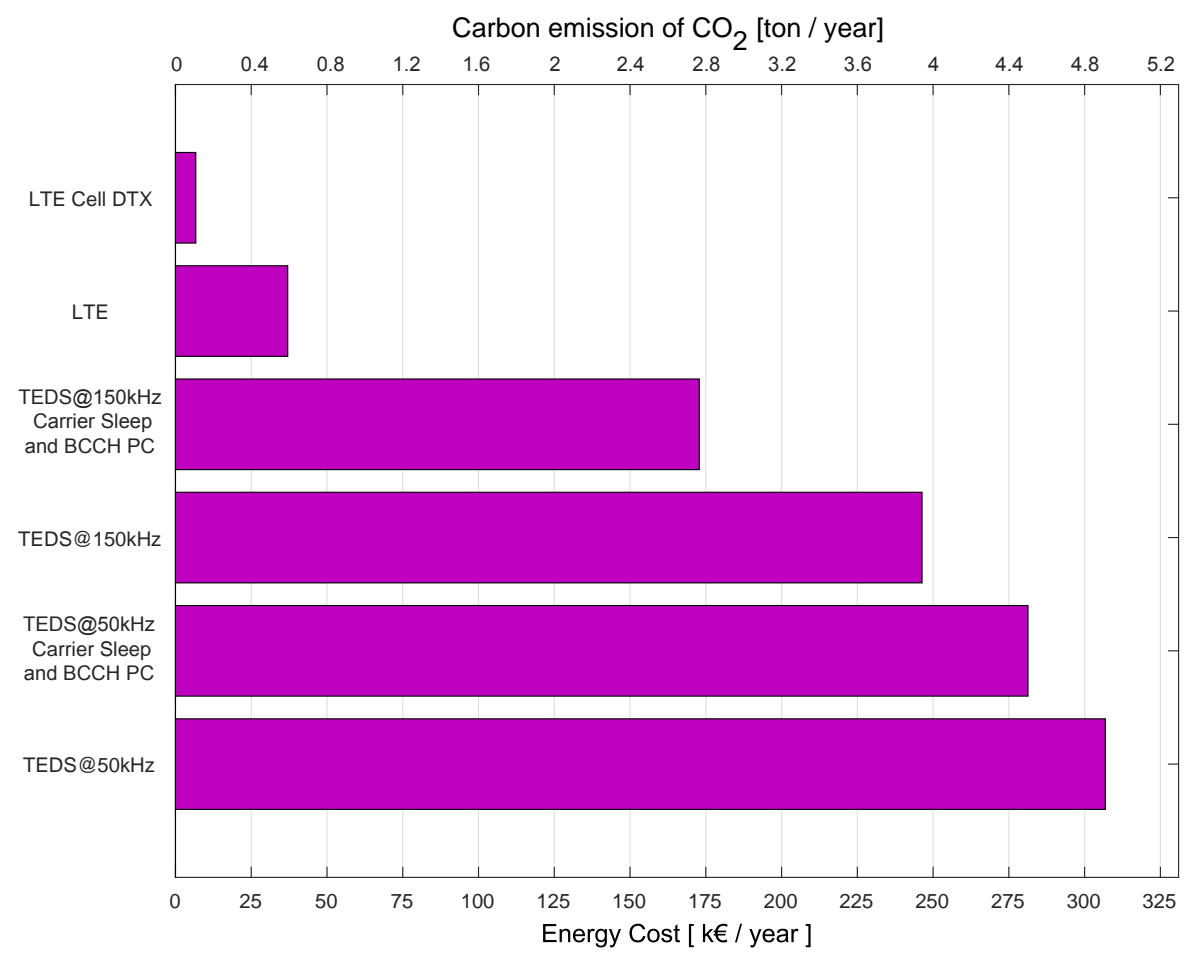

Figure 12. Annual energy expenditure and carbon emission of $\mathrm{CO}_{2}$ for TETRA and LTE networks.

\section{Conclusions}

In this paper, the energy efficiency of PMR systems has been considered. First, the most common PMR system, namely TETRA, has been introduced. After a discussion on the possibility of TETRA system to converge over the LTE platform, a power consumption model for a generic TETRA base station has been proposed. Then, the most effective energy saving solutions have been introduced and evaluated. In particular, the combined adoption of carrier sleep mode and $\mathrm{BCCH}$ power control ensures the reduction of TETRA base station baseline power consumption. The future transition to a TETRA over LTE system has then been considered, evaluating the impact of the most promising energy efficiency solutions on a deployed TETRA regional network. Results show significant improvements in terms of capacity and energy efficiency, as well as a positive impact in terms of OPEX and carbon footprint.

Author Contributions: Simone Morosi conceived and designed the experiments; Pierpaolo Piunti analyzed the data; Marco Dolfi performed the experiments and wrote the paper; Enrico Del Re organized and refined the manuscript. All authors have read and approved the final manuscript.

Conflicts of Interest: The authors declare no conflict of interest.

\section{References}

1. TETRA and Critical Communications Association. The Strategic Case for Mission Critical Mobile Broadband-A Review of the Future Needs of the Users of Critical Communications; White Paper; TETRA and Critical Communications Association: Newcastle upon Tyne, UK, 2013.

2. Gruet, C.; Pons-Masbernat, X.; Force, P. The LTE Evolution: Private Mobile Radio Networks. IEEE Veh. Technol. Mag. 2013, 8, 64-70.

3. Lasanen, M.; Aubree, M.; Cassan, C.; Conte, A.; David, J.; Elayoubi, S.E.; Galkin, T.; Grigore, V.; Le Masson, S.; Lees, J.; et al. Environmental friendly mobile radio networks: Approaches of the European OPERA-Net 2 project. In Proceedings of the 20th International Conference on Telecommunications (ICT), Casablanca, Morocco, 6-8 May 2013; pp. 1-5. 
4. Meo, M.; Zhang, Y.; Hu, Y.; Idzikowski, F.; Budzisz, L.; Ganji, F.; Haratcherev, I.; Conte, A.; Cianfrani, A.; Chiaraviglio, L.; et al. The TREND experimental activities on green communication networks. In Proceedings of the 24th Tyrrhenian International Workshop on Digital Communications-Green ICT (TIWDC), Genoa, Italy, 23-25 September 2013; pp. 1-6.

5. Olsson, M.; Fehske, A.; Hevizi, L.; Blume, O.; Vidacs, A.; Godor, I.; Fazekas, P.; Imran, M.; Qi, Y. Integration strategy of EARTH energy efficiency enablers. In Proceedings of the Future Network Mobile Summit (FutureNetw), Berlin, Germany, 4-6 July 2012; pp. 1-8.

6. Olsson, M.; Cavdar, C.; Frenger, P.; Tombaz, S.; Sabella, D.; Jantti, R. 5GrEEn: Towards Green 5G mobile networks. In Proceedings of the IEEE 9th International Conference on Wireless and Mobile Computing, Networking and Communications (WiMob), Lyon, France, 7-9 October 2013; pp. 212-216.

7. Bianco, C.; Cucchietti, F.; Griffa, G. Energy consumption trends in the next generation access network, a telco perspective. In Proceedings of the 29th International Telecommunications Energy Conference (INTELEC), Rome, Italy, 30 September-4 October 2007; pp. 737-742.

8. Davaslioglu, K.; Ayanoglu, E. Quantifying Potential Energy Efficiency Gain in Green Cellular Wireless Networks. IEEE Commun. Surv. Tutor. 2014, 16, 2065-2091.

9. Morosi, S.; Piunti, P.; Del Re, E. Improving cellular network energy efficiency by joint management of sleep mode and transmission power. In Proceedings of the 24th Tyrrhenian International Workshop on Digital Communications-Green ICT (TIWDC), Genoa, Italy, 23-25 September 2013; pp. 1-6.

10. Chen, T.; Yang, Y.; Zhang, H.; Kim, H.; Horneman, K. Network energy saving technologies for green wireless access networks. IEEE Wirel. Commun. 2011, 18, 30-38.

11. Feng, D.; Jiang, C.; Lim, G.; Cimini, L.J.J.; Feng, G.; Li, G. A survey of energy-efficient wireless communications. IEEE Commun. Surv. Tutor. 2013, 15, 167-178.

12. Wu, J.; Zhou, S.; Niu, Z. Traffic-Aware Base Station Sleeping Control and Power Matching for Energy-Delay Tradeoffs in Green Cellular Networks. IEEE Trans. Wirel. Commun. 2013, 12, 4196-4209.

13. Del Re, E.; Morosi, S.; Piunti, P.; Mazzi, G.; Gremigni, O. Energy efficient RRM strategies for current and upcoming TeTRA cellular systems. In Proceedings of the IEEE 10th International Conference on Wireless and Mobile Computing, Networking and Communications (WiMob), Lyon, France, 8-10 October 2014; pp. 40-44.

14. Ketterling, H.P.A. Introduction to Digital Professional Mobile Radio; Artech House, Inc.: Norwood, MA, USA, 2004.

15. European Telecommunications Standards Institute. Terrestrial Trunked Radio (TETRA); Voice plus Data $(V+D)$; Designers' Guide; Part 1: Overview, Technical Description and Radio Aspects; ETSI Technical Report 300-1; ETSI: Sophia Antipolis, France, 1997.

16. European Telecommunications Standards Institute. Terrestrial Trunked Radio (TETRA); Voice plus Data $(V+D)$; Part 2: Air Interface; ETSI Technical Report 392-2; ETSI: Sophia Antipolis, France, 2001.

17. Nouri, M.; Lottici, V.; Reggiannini, R.; Ball, D.; Rayne, M. TEDS: A high speed digital mobile communication air interface for professional users. IEEE Veh. Technol. Mag. 2006, 1, 32-42.

18. Doumi, T.; Dolan, M.; Tatesh, S.; Casati, A.; Tsirtsis, G.; Anchan, K.; Flore, D. LTE for public safety networks. IEEE Commun. Mag. 2013, 51, 106-112.

19. Ulema, M.; Kaplan, A.; Lu, K.; Amogh, N.; Kozbe, B. Critical communications and public safety networks, part 2: Technical issues, security, and applications. IEEE Commun. Mag. 2016, 54, 14-15.

20. Ferrus, R.; Sallent, O. Extending the LTE/LTE-A Business Case: Mission- and Business-Critical Mobile Broadband Communications. IEEE Veh. Technol. Mag. 2014, 9, 47-55.

21. 3rd Generation Partnership Project. Feasibility Study for Proximity Services (ProSe); 3GPP TR 22.803; 3GPP: Sophia Antipolis, France, 2014.

22. 3rd Generation Partnership Project. Study on Architecture Enhancements to Support Group Communication System Enablers for LTE (GCSE LTE); 3GPP TR 23.768; 3GPP: Sophia Antipolis, France, 2014.

23. 3rd Generation Partnership Project. Study on LTE Device to Device Proximity Services; Radio Aspects; 3GPP TR 36.843; 3GPP: Sophia Antipolis, France, 2014.

24. 3rd Generation Partnership Project. Study on Isolated Evolved Universal Terrestrial Radio Access Network (E-UTRAN) Operation for Public Safety; 3GPP TR 22.897; 3GPP: Sophia Antipolis, France, 2014.

25. 3rd Generation Partnership Project. Study on Radio Access Network (RAN) Sharing Enhancements; 3GPP TR 22.852; 3GPP: Sophia Antipolis, France, 2014. 
26. 3rd Generation Partnership Project. Public Safety Broadband High Power User Equipment (UE) for Band 14; 3GPP TR 36.837; 3GPP: Sophia Antipolis, France, 2007.

27. 3rd Generation Partnership Project. Enhancements for Multimedia Priority Service (MPS); 3GPP TR 23.854; 3GPP: Sophia Antipolis, France, 2012.

28. Auer, G.; Giannini, V.; Desset, C.; Godor, I.; Skillermark, P.; Olsson, M.; Imran, M.; Sabella, D.; Gonzalez, M.; Blume, O.; et al. How much energy is needed to run a wireless network? IEEE Wirel. Commun. 2011, 18, 40-49.

29. TETRA and Critical Communications Association. Mobile Broadband for Critical Communications Users-A Review of Options For Delivering Mission Critical Solutions; Technical Report; TETRA and Critical Communications Association: Newcastle upon Tyne, UK, 2013.

30. Auer, G.; Blume, O.; Giannini, V.; Godor, I.; Imran, M.; Jading, Y.; Katranaras, E.; Olsson, M.; Sabella, D.; Skillermark, P.; et al. D2.3: Energy Efficiency Analysis of the Reference Systems, Areas of Improvements And Target Breakdown; INFSOICT-247733 EARTH (Energy Aware Radio and neTwork tecHnologies), Tech. Rep; 2010. Available online: http:/ / cordis.europa.eu/docs/projects/cnect/3/247733/080/deliverables / 001-EARTHWP2D23v2.pdf (accessed on 23 December 2016).

31. Tombaz, S.; wook Han, S.; Sung, K.W.; Zander, J. Energy Efficient Network Deployment With Cell DTX. IEEE Commun. Lett. 2014, 18, 977-980.

(C) 2016 by the authors; licensee MDPI, Basel, Switzerland. This article is an open access article distributed under the terms and conditions of the Creative Commons Attribution (CC-BY) license (http:/ / creativecommons.org/licenses/by/4.0/). 